BNL-113553-2017-JA

\title{
A Software for managing afterhours activities in research user facilities
}

\author{
Fernando E. Camino
}

Submitted to the Journal of Chemical Health and Safety

October 2016

Center for Functional Nanomaterials

Brookhaven National Laboratory

\author{
U.S. Department of Energy \\ USDOE Office of Science (SC), \\ Basic Energy Sciences (SC-22)
}

\footnotetext{
Notice: This manuscript has been authored by employees of Brookhaven Science Associates, LLC under Contract No. DE- SC0012704 with the U.S. Department of Energy. The publisher by accepting the manuscript for publication acknowledges that the United States Government retains a non-exclusive, paid-up, irrevocable, world-wide license to publish or reproduce the published form of this manuscript, or allow others to do so, for United States Government purposes.
} 


\section{DISCLAIMER}

This report was prepared as an account of work sponsored by an agency of the United States Government. Neither the United States Government nor any agency thereof, nor any of their employees, nor any of their contractors, subcontractors, or their employees, makes any warranty, express or implied, or assumes any legal liability or responsibility for the accuracy, completeness, or any third party's use or the results of such use of any information, apparatus, product, or process disclosed, or represents that its use would not infringe privately owned rights. Reference herein to any specific commercial product, process, or service by trade name, trademark, manufacturer, or otherwise, does not necessarily constitute or imply its endorsement, recommendation, or favoring by the United States Government or any agency thereof or its contractors or subcontractors. The views and opinions of authors expressed herein do not necessarily state or reflect those of the United States Government or any agency thereof. 


\title{
A Software for managing afterhours activities in research user facilities
}

\author{
F E Camino \\ Center for Functional Nanomaterials, Brookhaven National Laboratory, Upton, New \\ York 11973
}

We present an afterhours activity management program for shared facilities, which handles the processes required for afterhours access (request, approval, extension, etc.). It implements the concept of permitted afterhours activities, which consists of a list of well-defined activities that each user can perform afterhours. The program provides an easy and unambiguous way for users to know which activities they are allowed to perform afterhours. In addition, the program can enhance its safety efficacy by interacting with lab and instrument access control systems commonly present in user facilities.

\section{Introduction}

For several reasons, e.g., to maximize the usage of high-demand instrumentation, research user facilities need to allow access to instrumentation and laboratories during times when permanent staff is not present, commonly known as afterhours access. For this purpose, the institution gives some users the privilege, for a finite period of time, to work afterhours based on the users' experience and on the type of afterhours activities. For medium to large shared facilities, the management of afterhours access can become quite burdensome and intricate. It requires to handle processes such as requesting afterhours access, gathering approvals from staff and safety personnel, notifying users of approval, monitoring the time during which access is valid, and requesting and approving extensions of afterhours access, among several other processes which are performed manually, mostly via extensive use of emails. Moreover, the creation and maintenance of an afterhours user database is not automatic. As a result, on one hand, basic information for staff - like the number of afterhours users in a facility, or the number of facilities that a user can access - is not readily available. On the other hand, users do not have a simple way to know which facilities they can access and which activities they can perform afterhours.

In view of the problems just described, we have created a computer program that manages the processes required for afterhours access (request, approval, extension, etc.) and automatizes the creation and maintenance of an afterhours database. The software implements the safety concept of permitted afterhours activity: each facility has a list of well-defined afterhours activities that users can perform. This concept and the afterhours managing software allow: 1) staff and safety personnel to have clear criteria when evaluating afterhours requests, and 2) users to know unambiguously which activities they are allowed to perform afterhours.

The program is to be implemented in the Center for Functional Nanomaterials (CFN) at Brookhaven National Laboratory in New York. Similar user facilities can modify the software to fit their particular operations or, alternatively, can build their own applications based on the safety concepts and the structure of the software presented here. ${ }^{1}$ 
Section 2 gives an overview of the afterhours request and approval process in the CFN, which is implemented by the afterhours management software described in Section 3. Section 4 discusses the additional safety benefits of the afterhours software when it is configured to interact with lab and instrument access control systems usually implemented in user facilities.

\section{Afterhours work policy in a multi-user facility: Example of the Center for Functional Nanomaterials (CFN)}

The CFN is a user-oriented research center that serves the nanoscience research community. It hosts unique state-of-the-art instrumentation and facilities which are highly subscribed, and which, consequently, require to be accessed afterhours in order to maximize the benefit they offer to the user community. For example, CFN's dedicated electron lithography tool totaled about 5,000 hours of afterhours use in fiscal year 2015. ${ }^{2}$ Afterhours work poses two main types of potential hazardous situations: a) personal health hazards when accessing facilities that host hazardous chemicals and equipment (e.g., when accessing the nanofabrication or materials synthesis facilities), and b) potential damage to costly instrumentation due to improper use. To minimize the chance of accidents during afterhours work, the CFN has established a protocol for requesting and approving afterhours work, which has the following main elements.

\subsection{List of permitted afterhours activities}

At the heart of the protocol is the CFN list of permitted afterhours activities, which encompasses all the processes that can be performed afterhours in the CFN. Each afterhours activity in the list is "owned" by a scientific staff, referred as "staff" in this manuscript, who is the most knowledgeable person regarding the technical aspects of the activity. In addition, each activity in the list has been reviewed and approved by the environmental, safety and health staff (referred as "ES\&H staff" in the manuscript), who, by virtue of the training received, has the final word in deciding whether an activity is safe to be performed afterhours.

\subsection{How users add afterhours activities to their personal list of afterhours activities}

A user is any person performing research in the facility who is not a staff member. Each user possesses a personal list of permitted afterhours activities, which consists of a subset of the CFN list. A user requests a new activity to be included in his/her personal list from the staff owning the activity. If the staff considers that the user is able to perform the activity safely afterhours, the staff elevates the request to the ES\&H staff, who whether grants or denies the request.

\section{Description of the software}

\subsection{Main user interface}

Fig. 1 shows the main user interface. It is divided into four horizontal sections: User information, Approved facilities, Requested activities and Staff review. Users utilize the main user interface to view the activities that they can perform afterhours, to request renewals of existing activities and to request new afterhours activities for consideration by staff and safety personnel. Staff also use the interface to access administrative sections of the program where they can, for example, approve user requests and add (or remove) afterhours activities. 


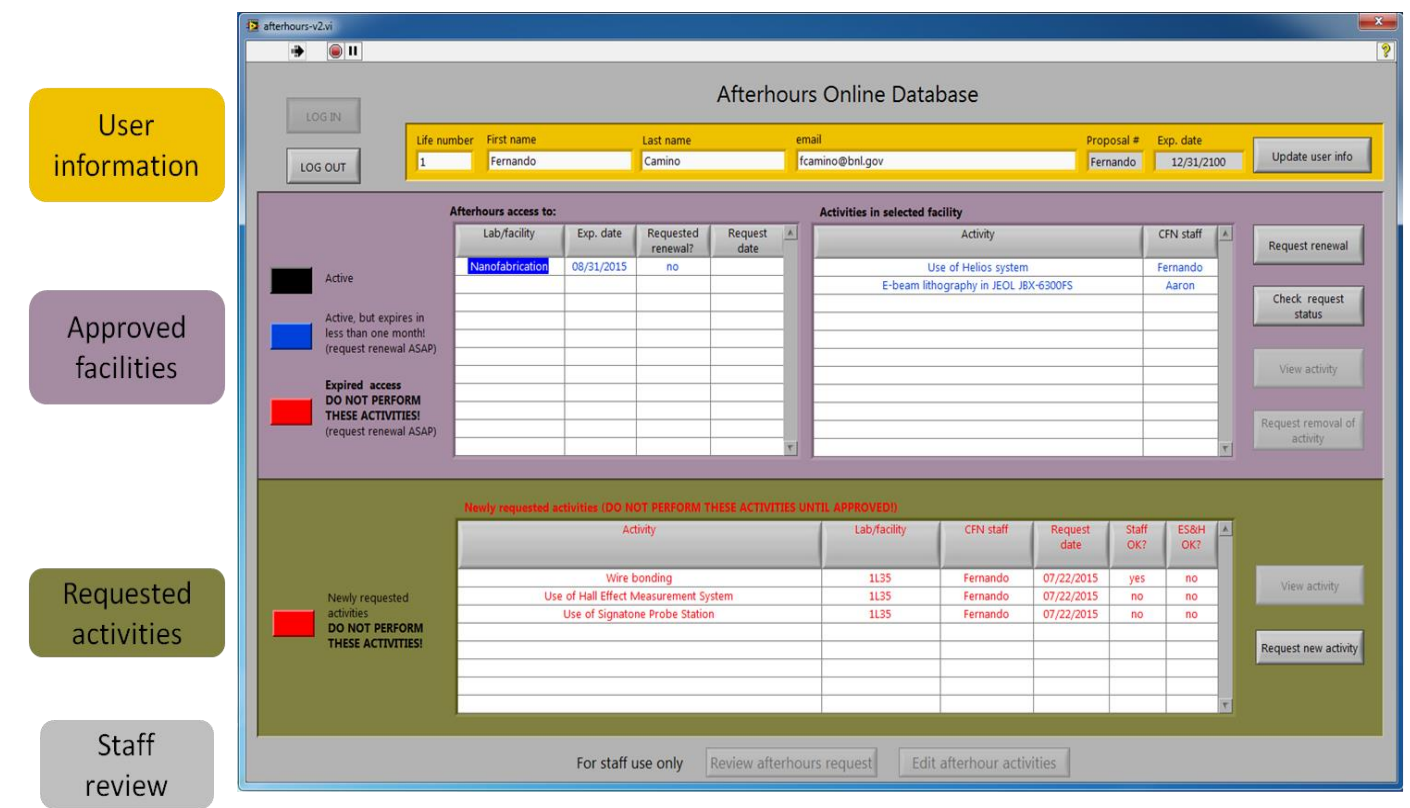

Figure 1. Main user interface. The horizontal sections are: User information (yellow), Approved facilities (purple), Requested activities (olive) and Staff review (gray).

3.1.1. User information section. This section allows users to log in the afterhours software using a single ID (life number). The software validates a login by retrieving user-related information from a database maintained by a stand-alone facility login program (not described in this article). ${ }^{3}$

3.1.2. Approved facilities section. In this section users can review which facilities they can access afterhours, and more importantly, users can verify which activities they can perform in a selected facility (Fig. 2a). Here is where the concept of permitted afterhours activities is implemented. ${ }^{4}$ During training, users are instructed to perform only the unexpired activities listed in this section. Also, users are aware that performing any unlisted or expired activities is a severe safety violation, which triggers harsh disciplinary actions. Section 4 will further discuss the safety aspects of the afterhours software.

a) Selected facility

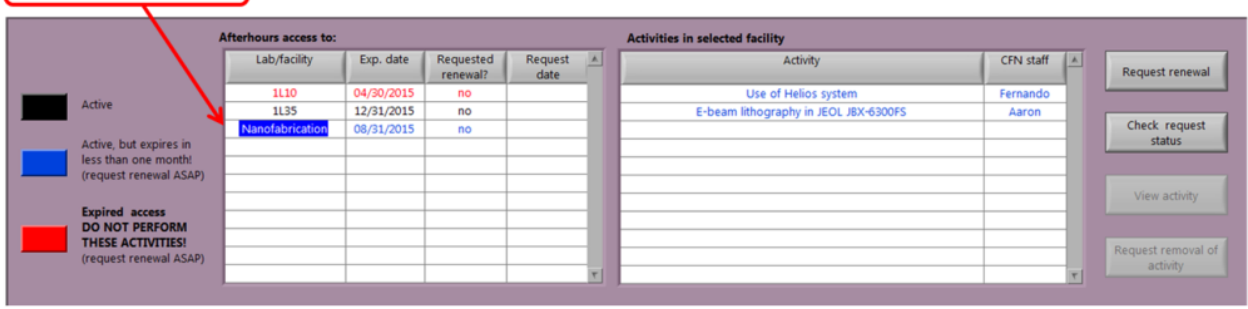

b)



Figure 2. a) Approved activities section. The left panel lists the facilities that a given user can access afterhours (the second column is the facility access expiration date). The right panel lists the permitted activities in the selected facility. Four commands are displayed at the rightmost section. b) Approval status table for the facility selected in (a), which pops up when the user clicks on the "Check request status" button. This table is updated in real time. 
Approved afterhours activities are grouped by the facility in which they are performed. This allows the program to process renewal of facility access instead of processing numerous renewals for each activity in a facility. This is important since it streamlines the renewal process for staff and users without compromising safety. Users request 1) access to new afterhours activities and 2) renewal of previously approved facility access. The process of requesting new activities is discussed in Section 3.2.2. This section focuses on the renewal of pre-approved facility access.

Each facility needs to define the period of validity of afterhours requests. For example, in the CFN, this period is four months (Jan-Apr, May-Aug and Sep-Dec). The following description assumes, as a practical example, a period of validity of four months. Expired facility access can be renewed at any time during a period. However, to extend valid facility access without interruptions from one period to the next, users need to request renewals sometime during the current period. To prevent users from requesting renewals randomly during a period, the program allows renewal of active facility access only during the last (fourth) month of the current period. During the first three months, renewal of active facility access is disabled (facilities in black font correspond to this situation, see Fig. 2a). At the beginning of the fourth month, the program automatically sends emails reminding users to renew their facility access and changes the font color of active facilities from black to blue to signal the need for renewal. Once a new period starts, unrenewed facility access expires and is displayed in red font (Fig. 2a). Users request renewal of active (blue font) or expired (red font) facilities by clicking on the "Request renewal" button (right most area in Fig. 2a). Users can also view the progress of their request by clicking on the "Check request status" button, which opens a pop-up window with real-time information of the renewal process (Fig. 2b). The facility access renewal process is described in detail in Section 3.2.1. There are two more commands, which are enabled only when a user selects an activity in the list displayed on the right panel in Fig. 2a. The "View activity" button displays the "activity form" of the selected activity (Fig. 3). The activity form is an electronic form generated and updated by the staff owning the activity and by the designated environmental, safety and health $(\mathrm{ES} \& \mathrm{H})$ staff. It contains the description of the activity, a list of equipment and chemicals used, and a list of hazardous procedures, among other useful safety information regarding the activity. The activity form is available to users for viewing and to staff for editing. (Section 3.3 describes the module used to edit or add afterhours activities in the system.) Finally, the "Request removal of activity" button notifies staff when a user does not require an afterhours activity any longer.

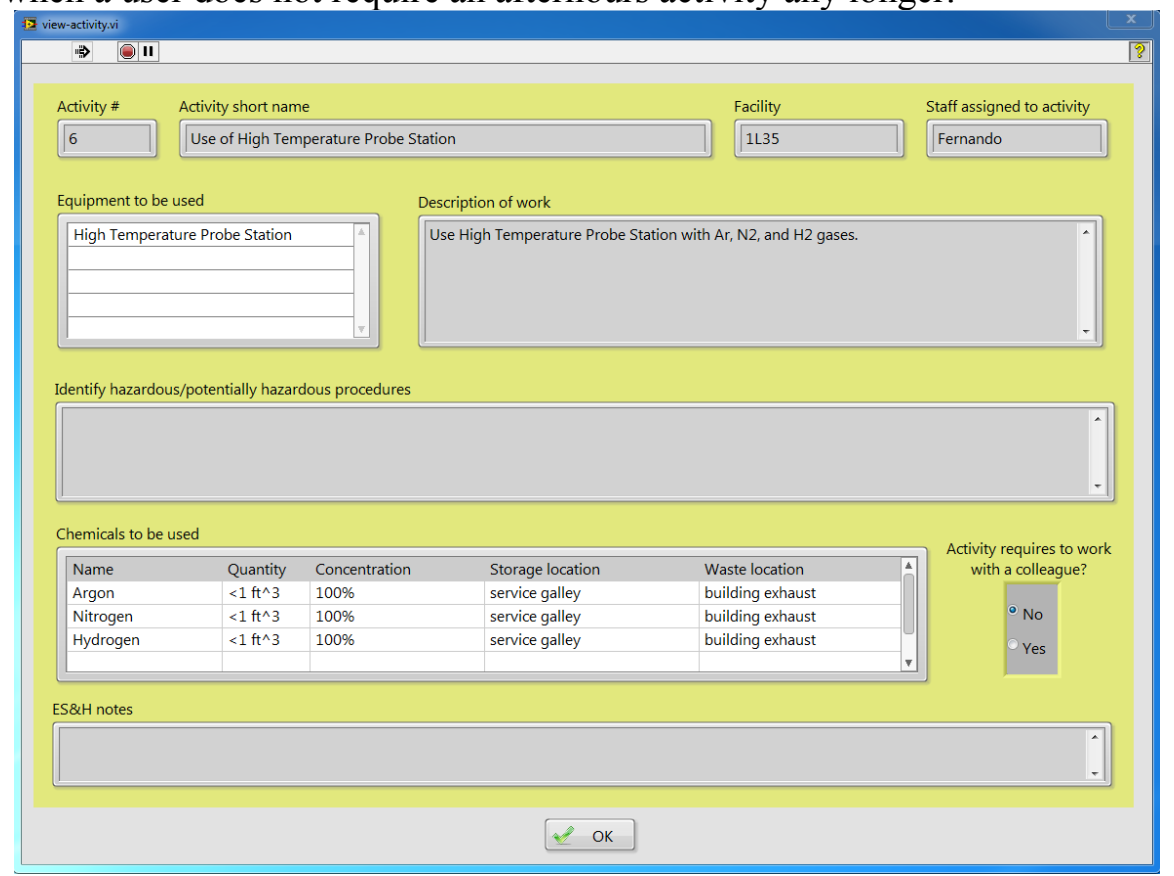

Figure 3. Example of and activity form displayed when a user clicks on the "View activity" button of the Approved facilities section of the user interface (see Fig. 2a). 
3.1.3. Requested activities section. The requested activities section of the main user interface lists all the new activities requested by a user (Fig. 4). Users request new activities by clicking on the "Request new activity" button, which launches the "Request new activity" module shown in Fig. 5. This module allows users to request one or more new afterhours activities. ${ }^{5}$ It consists of two sections: "activity selection" and "activities-to-be-requested" (pink and light blue areas in Fig. 5, respectively). The top right table in Fig. 5 lists the available activities in the facility selected in the top left table. Users can move a selected activity to the activities-to-be-requested table using the "Move to request list" button. The "Request ALL and exit" command transfers all the activities listed in the activitiesto-be-requested table to the requested activities section of the main user interface (Fig. 4), and also starts the new activity approval process, which is described in Section 3.2.2. Once the corresponding staff owning the activity (CFN staff in Fig. 4) and the ES\&H staff have approved a request, indicated by "yes" in the "Staff OK?" and "ES\&H OK?" columns in Fig. 4, the activity is added to its corresponding facility in the approved facilities section of the main user interface.

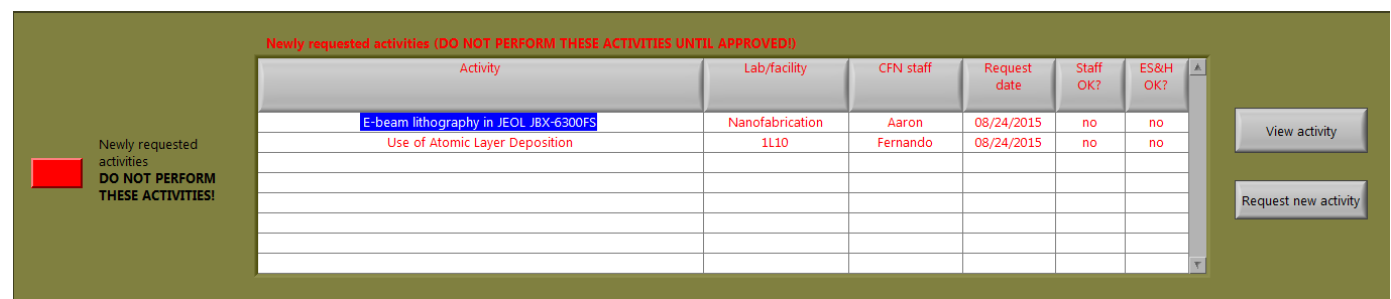

Figure 4. Requested activities section of the main user interface. Requested activities are listed in this section while the new activity request approval process is pending (Section 3.2.2). The "View activity" button displays the activity form (Fig. 3) of a selected activity.

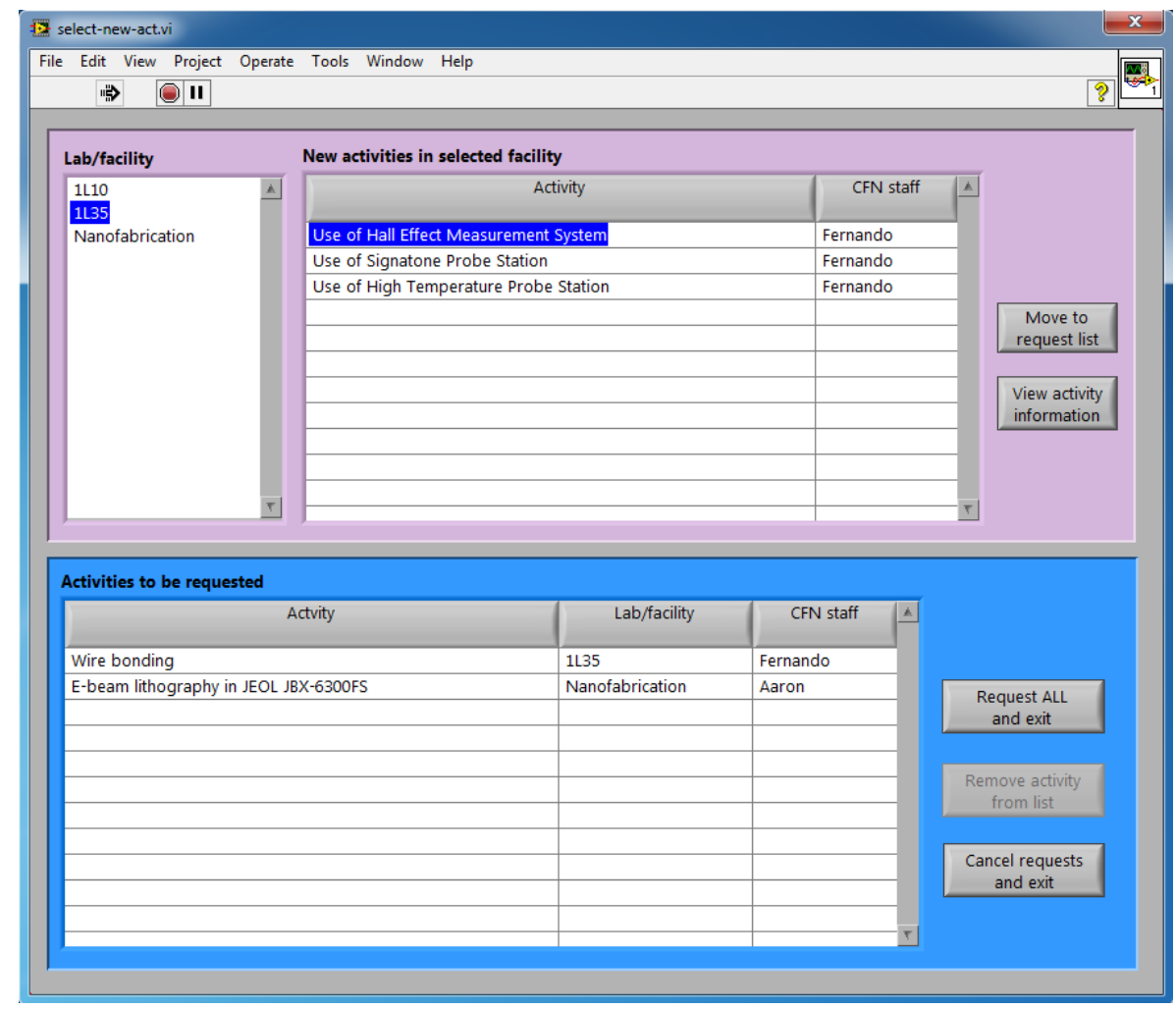

Figure 5. Request new activity module. Users can view the activity form (Fig. 3) of a selected activity with the "View activity information" button. The "Move to request list" button transfers a selected activity from the top to the bottom panel. Once all desired activities are transferred, the "Request ALL and exit" button starts the process of approval of new activities, which is explained in Section 3.2.2. 
3.1.4. Staff review section. This section is the last section of the main user interface and can only be accessed by staff. It consists of two buttons (see bottom section in Fig. 1). The "Review afterhours request" button launches the "Review afterhours request" module, which is used by staff to manage requests for renewal of facility access, as well as requests for new activities. This module is described in the next section. The second button, "Edit afterhours activities," launches the "Edit afterhours activities" module, which allows staff to edit and add activities (and associated activity forms) to the afterhours activities database. This module is described in Section 3.3.

\subsection{The review afterhours request module}

This module allows staff to process afterhours requests following the particular protocol established in the user facility. This description uses the protocol implemented by the Center for Functional Nanomaterials. Fig. 6 shows the module's user interface, which consists of four parts: 1) users list (leftmost list), 2) user information (olive area), 3) facility access (light green area), and 4) new activities (yellow area). The list of users and the corresponding user-related information (olive area) are obtained by querying the user database maintained by the facility login software. ${ }^{3}$ The facility access and the new activities sections are described in Sections 3.2.1 and 3.2.2, respectively.

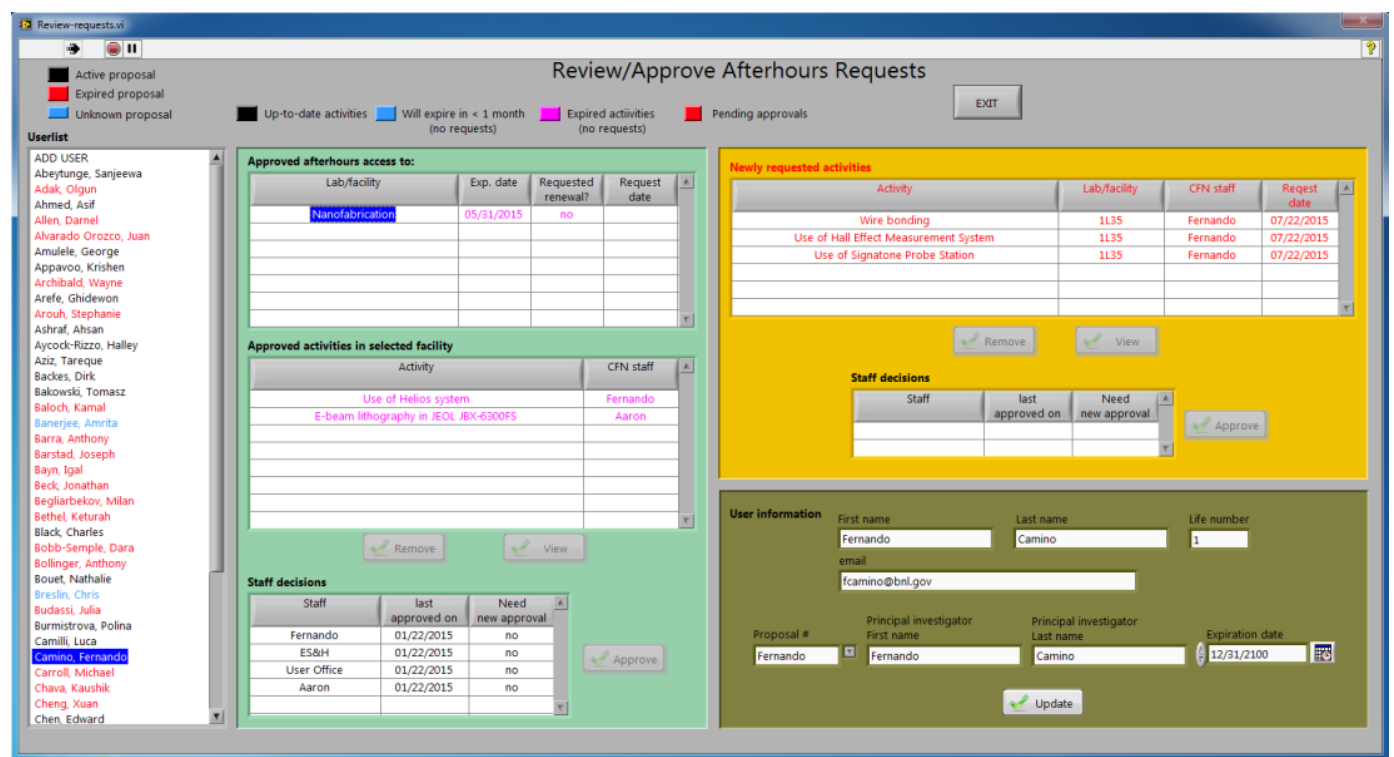

Figure 6. User interface of the review afterhours request module. Staff process requests for renewal of facility access in the facility access section (light green area). Requests for new afterhours activities are processed in the new activities section (yellow area).

3.2.1. Review afterhours request module: facility access renewal. When users request renewal of facility access (see Section 3.1.2), they initiate a multi-step process depicted in Fig. 7. This section describes this process step by step, using as an example the initial situation shown in Fig. 8a. 


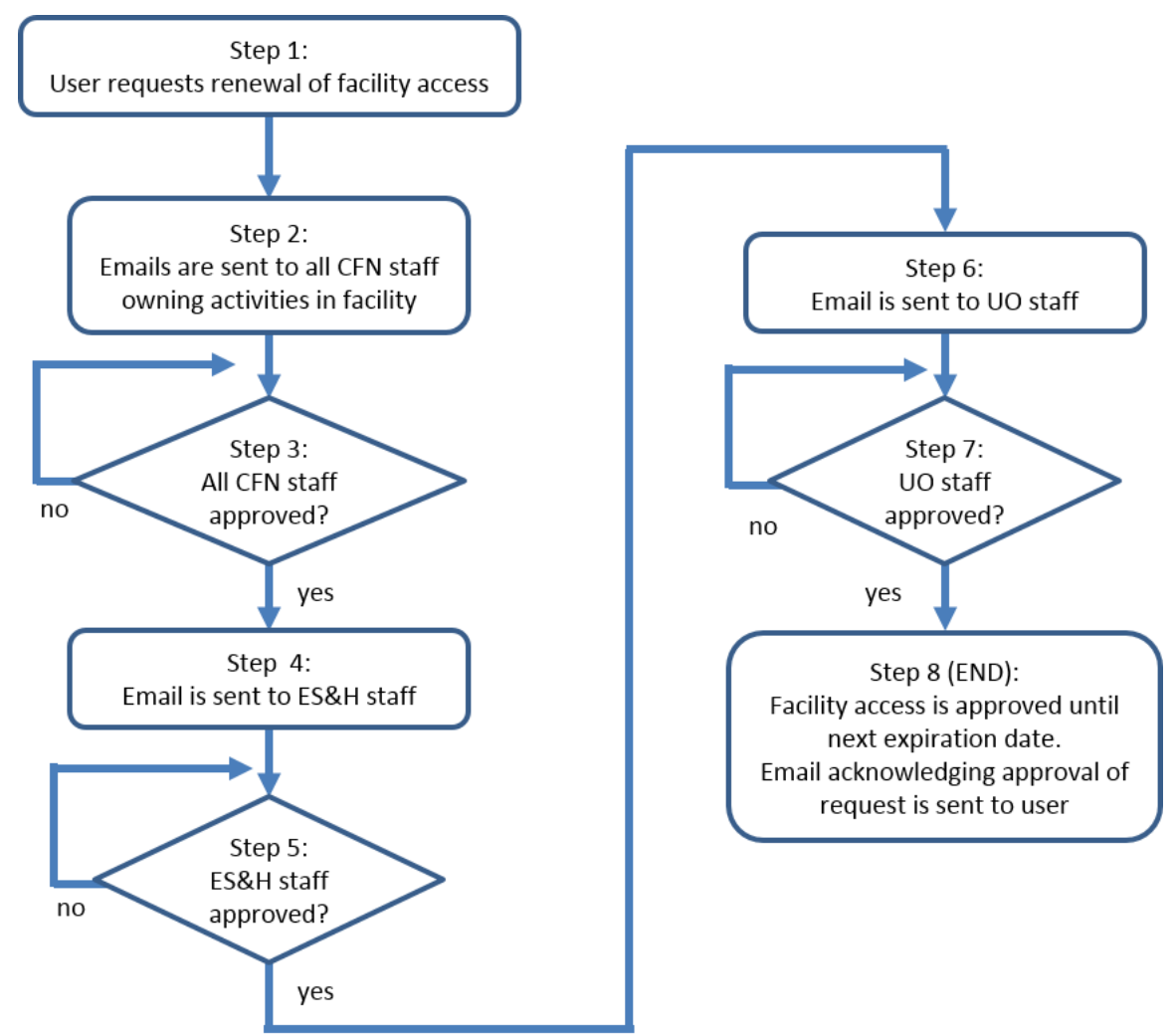

Figure 7. Process flow to approve a user request for renewal of facility access (UO stands for Users Office).

a)

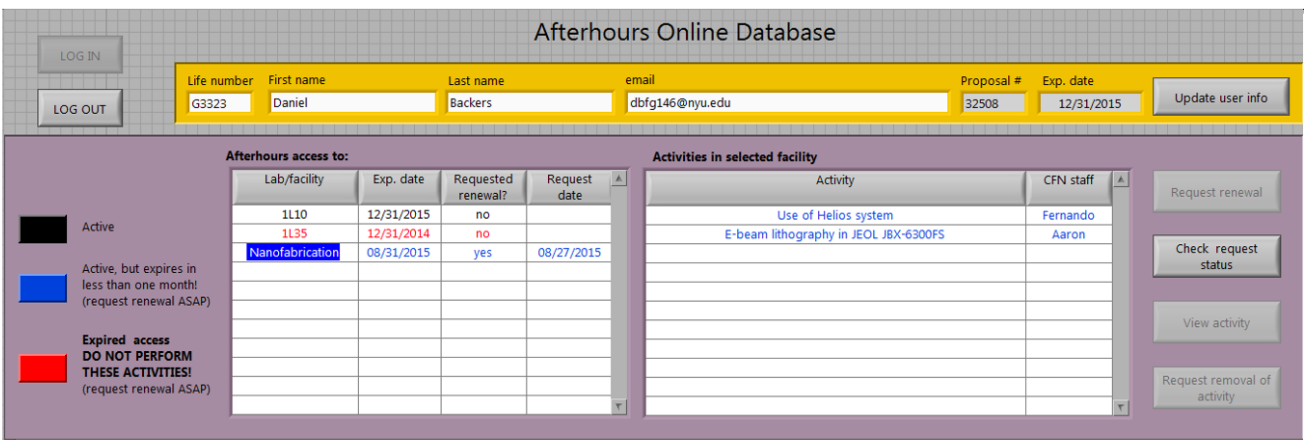

b) Subject: Afterhours facility access renewal request
The following user: Daniel Backers
Life \#: G3323
email:sdbfg146@nyu.edu
Proposal \# : 32508
is requesting renewal of afterhours access to facility: Nanofabrication.
Please go to the Online Afterhours System to review this request.

Figure 8. a) Example used to describe the steps involved in the processing of a request for renewal of facility access. The user selects a blue font facility (Nanofabrication) and clicks on the "Request renewal" command, which initiates the renewal process. b) Subject and message of the email sent to the staff in the right table in (a). 
- Steps 1 and 2: user requests renewal of facility access, followed by emails to staff. The process starts when the user selects a facility to renew and clicks the "Request renewal" button in the approved facilities section of the main user interface (Fig. 8a). In this example, the facility is active, but it is requiring renewal for the following period, since it is the last (fourth) month of the current period (activities and facilities in blue color denote this circumstance as explained in Section 3.1.2). The approval process is the same in case the requested facility is expired (facility and activities in red font). Fig. 8a shows that the selected facility contains two activities, which are owned by two staff: Fernando and Aaron. The "Request renewal" button automatically sends emails only to these two staff members. The information in the email (shown in Fig. 8b) allows staff to locate the request for review and approval in the facility access section of the review afterhours request module (Fig. 9).

- Steps 3 and 4: collect staff approvals, followed by email to ES\&H staff. Any staff can access the review afterhours request module (Figs. 6 and 9) to review user afterhours access; however, only staff owning activities in a requested facility are allowed to approve the user's request. Staff locate the requesting user from the module's user list (leftmost list in Fig. 9) and select the requested facility (nanofabrication in this example).

As shown in Fig. 9, the facility access section of the module (light green area) consists of three tables: the top table lists the user's active (and expired) facilities, the center table lists the activities in a selected facility and the bottom table shows the approval status for a selected facility. The contents of the top two tables are color coded to facilitate their identification. Black and blue fonts indicate active facilities in the first three months and last month of a current period, respectively (same font color coding as in the main user interface). Magenta font corresponds to expired facilities without renewal request, while red font corresponds to facilities with a pending request for renewal. Only facilities in red font require approval from staff. The middle table has two commands acting on a selected activity. The "Remove" button removes the activity from user list. Only the staff owning the activity can use this button, other staff members see this button disabled. However, any staff can use the "View" button, which displays the activity form (Fig. 3) of the selected activity. 


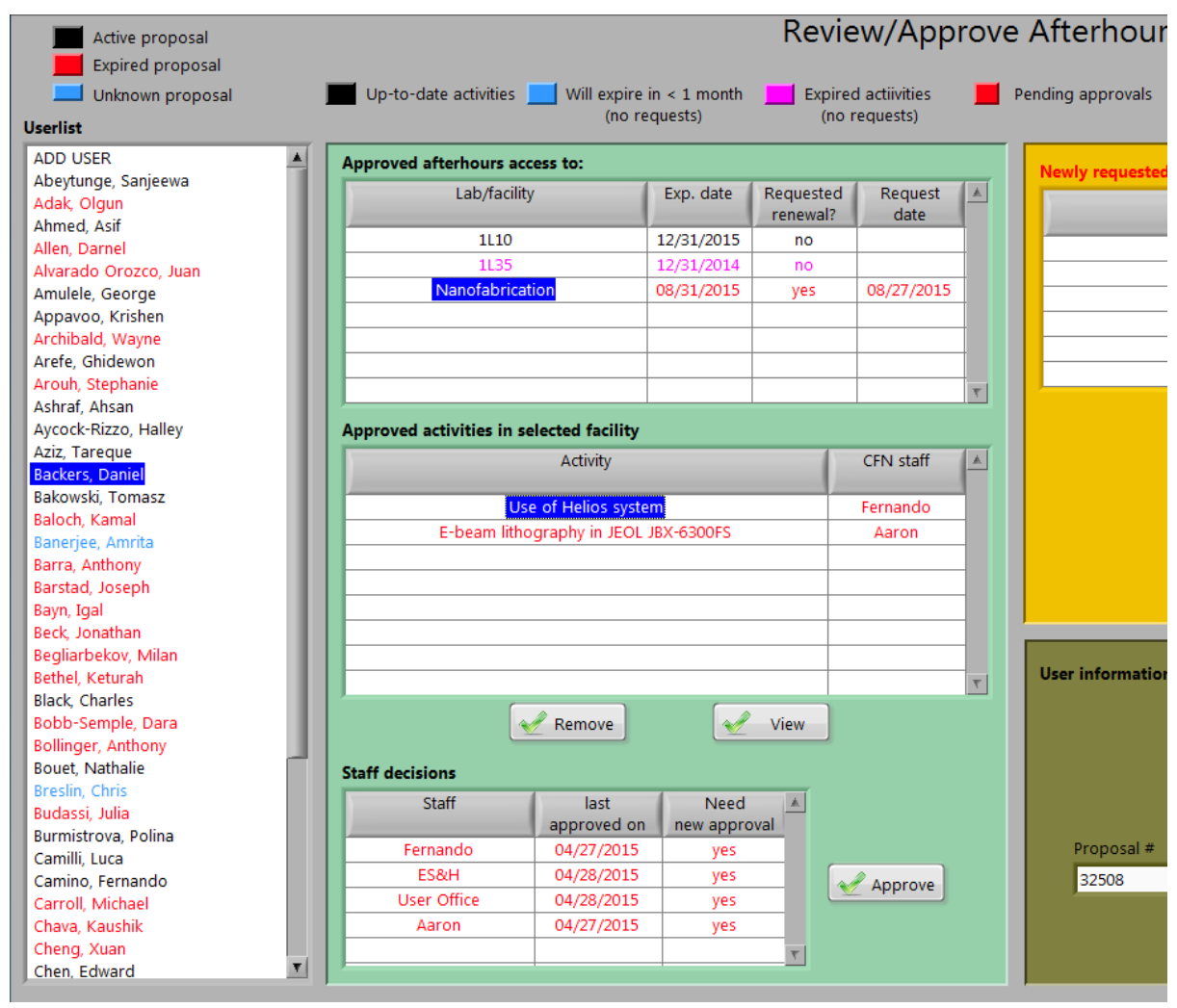

Figure 9. Partial view of the review afterhours request module showing the user list and facility access sections.

The bottom table in Fig. 9 lists the staff approval status for a selected facility. Its content is color coded: red (black) indicates that the staff is (is not) pending an approval. A facility is approved (active) when all staff listed in the approvals table - including ES\&H and User Office staff-have approved the request. In this example, at the present stage of the process (step 3), only staff owning activities in the selected facility (Fernando and Aaron) can approve the request, which is done with the "Approve" button next to the approval status table. Users have real time access to the approval process using the "Check request status" button in the approved facilities section of the main user interface (Fig. 8a). Fig 10 shows the corresponding approval status tables for the main user interface (seen by users) and review afterhours request module (seen by staff). The tables indicate that Fernando and Aaron have approved the request. This situation activates step 4 of the approval process: send email to ES\&H staff. The email content is the same as in Fig. 8b, its purpose is to alert the ES\&H staff that there is a request awaiting approval.

a)

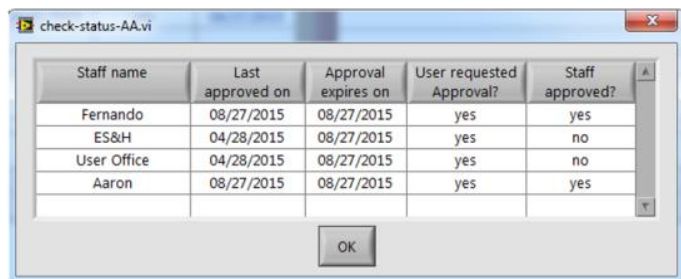

b)

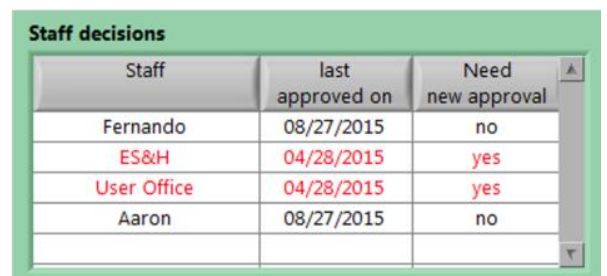

Figure 10. Request approval status tables in (a) main user interface (seen by users) and (b) review afterhours request module (seen by staff). Both tables indicate that the staff owning requested activities (Fernando and Aaron) have approved the request. 
- Steps 5 and 6: collect ES\&H staff approval, followed by email to Users Office staff. Once the ES\&H staff approves the request, the approval status tables are updated and an email (Fig. 11a) is sent to the Users Office staff, whose approval finalizes the request approval process. In the Center for Functional Nanomaterials, the Users Office staff is in charge of encoding the user's identification badge for afterhours access to the requested facility. ${ }^{6}$

- Steps 7 and 8: collect Users Office staff approval and set facility access active. After the Users Office staff has approved the request, the approvals tables are finally updated and the program sends an email to the user acknowledging approval of the facility renewal request (Fig. 11b). The expiration date depends whether the last approval was performed during the last (fourth) month or during the first three months of a period. In the former case, as in this example, the new expiration date is the end of the following period. In the latter case, the expiration date is the end of the current period. Fig. 11c shows the final status of the facility access section of the program.

a)

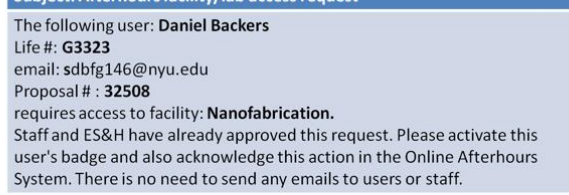

b)

Subject: Afterhours facility/lab access renewal approved
We have renewed your afterhours access to facility:
Nanofabrication.
Please go to the Online Afterhours System to see the
activities you are allowed to perform.

c)

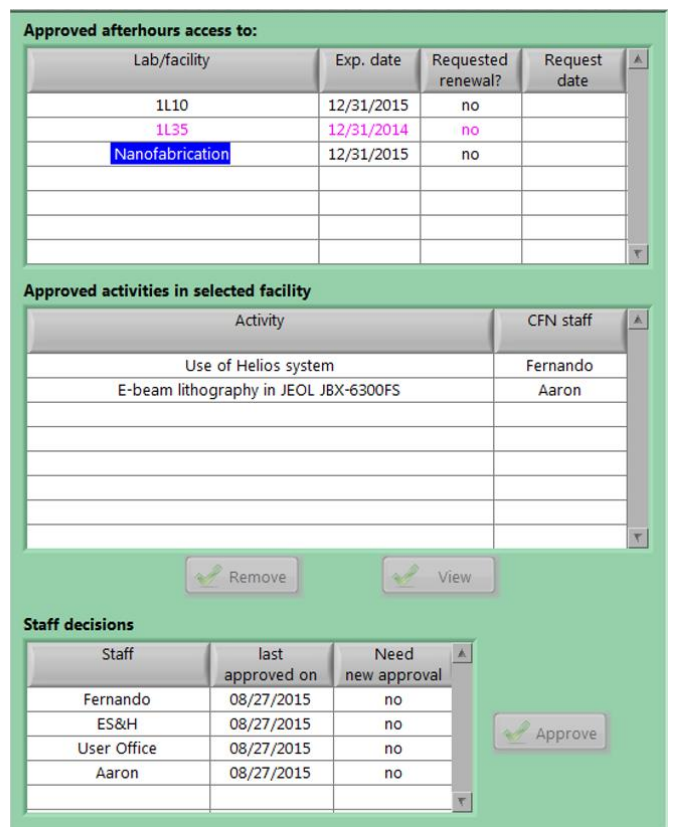

Figure 11. Final steps of the facility request approval process. a) Email sent to the Users Office staff. b) Email sent to the user acknowledging approval of request. c) Final status of the facility access section of the review afterhours request program.

\subsubsection{Review afterhours request program: Approval of requests of new activities}

The steps for approving requests of new afterhours activities (Fig. 12) differ slightly from the steps for renewing facility access described in the previous section. Requests for new activities are handled in the new activities section of the review afterhours request module (Figs. 6 and 13b).

- Steps 1 and 2: user requests new activities, followed by emails to staff. The process for approving new activities starts when users click the "Request ALL and exit" button of the request new activity module (Fig. 5). Let us use the situation shown in Fig. 5 as an example. As explained in section 3.1.3, the "Request ALL and exit" button transfers all the activities in the bottom table of Fig. 5 to the requested activities section of main user interface, which is shown in Fig. 13a. Note that this table already had a previously requested new activity with pending request, namely, "Use of atomic Layer Deposition." The "Request ALL and exit" button also triggers emails to all staff owning requested activities. 


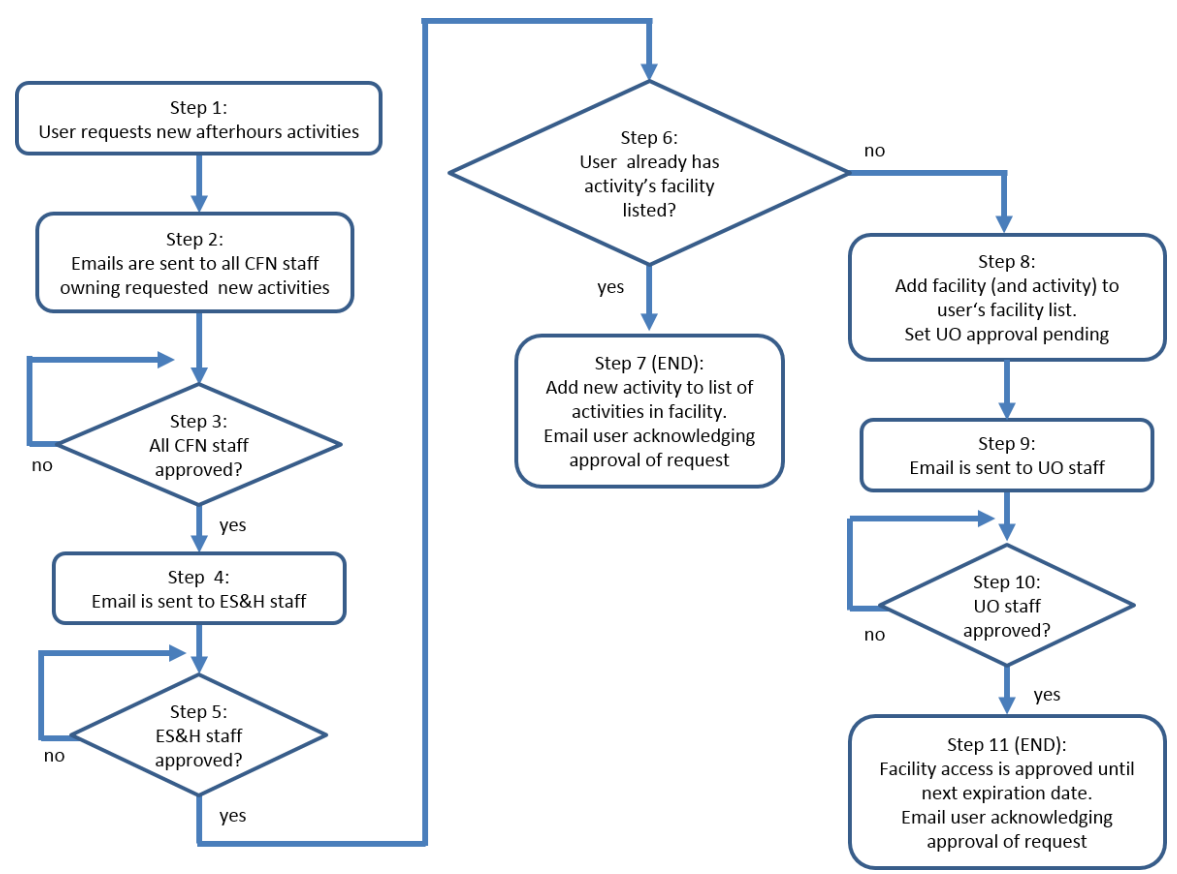

Figure 12. Steps to approve a user request for new afterhours activities (UO stands for Users Office).

a)

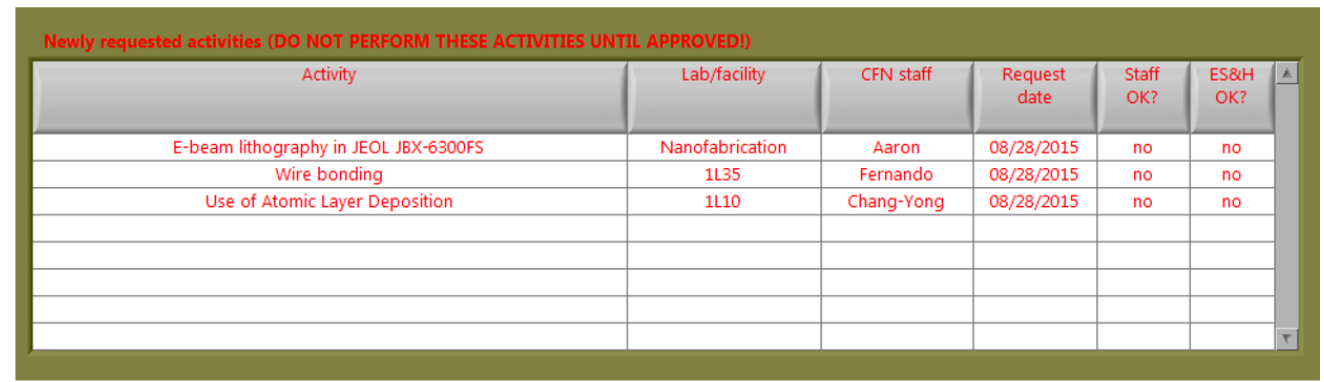

b)

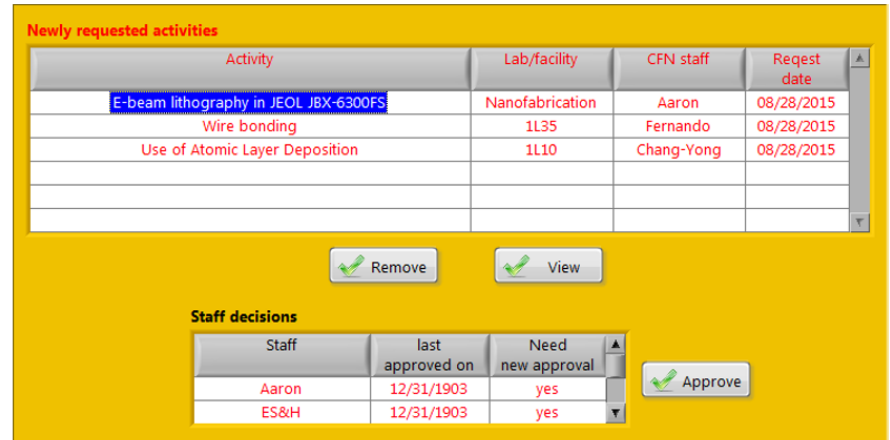

Figure 13. a) Requested activities section of the main user interface after the user has requested the two activities shown in the bottom table of Fig. 5. Notice that there was already one activity in this table from a previous request. b) New activities section of the review afterhours request module. Here, Aaron has selected the activity, but has not yet clicked the "Approve" button.

- Steps 3 and 4: collect staff approval, followed by an email to ES\&H staff. Staff use the review afterhours request module to process the requested new activities, which are listed in the new activities section (top table in Fig. 13b). The middle area of Fig. 13b shows two buttons, which act on a selected activity: the "Remove" button allows the staff owning the selected activity to erase it from the user's list, and the "View" button displays the activity form (Fig. 3). The bottom table in Fig. 13b is the approval status table of the selected activity. 
It is color coded, with red and black font indicating pending and completed approvals, respectively. In this example, Aaron has accessed the module. Once he clicks the "Approve" button, the tables in Fig. 13 will be updated and an email will be sent to the ES\&H staff.

- Steps 5 and 6: collect ES\&H staff approval, and determine if the user has the activity's facility already listed. Fig. 14a shows the review afterhours request module after the ES\&H staff has selected the activity approved by Aaron in Step 3. Once the ES\&H staff clicks the "Approve" button, the approval process takes different paths depending on whether the facility of the selected activity, nanofabrication in this case, is or is not listed in the facility access section of the module. Step 7 considers the case where the selected activity's facility is listed, as shown in Fig. 14a. Steps 8 through 11 describe the alternative situation.

- Step 7 (Facility is listed): add requested activity to list of activities in facility and email user acknowledging approval. This step is the final stage of the approval process, provided that the user has access to the facility of the requested activity. As shown in Fig. 14b, the requested activity is automatically added to the nanofabrication facility activities (central table in light green area) and is erased from the new activities section (yellow area). Next, the nanofabrication facility approval status table (bottom table in light green area) is updated. Finally, an approval acknowledgement email is sent to the user.

a)

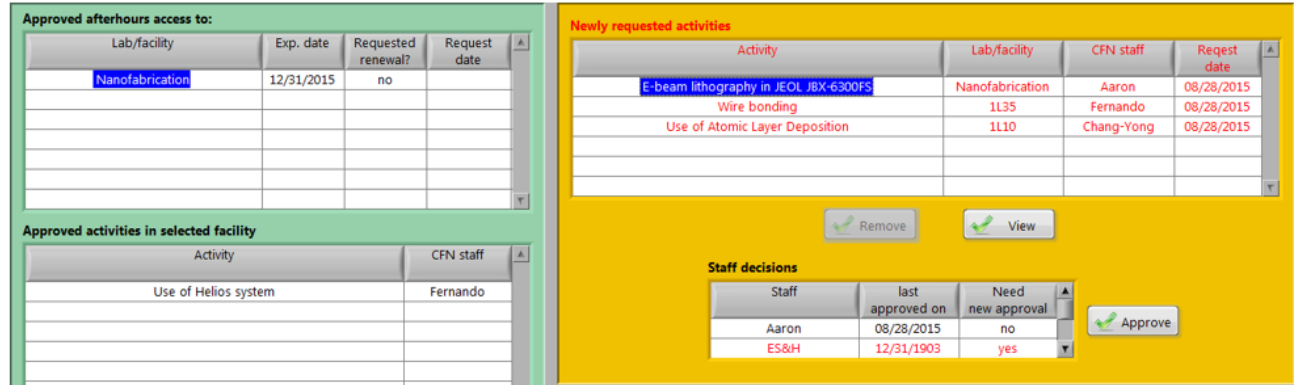

b)

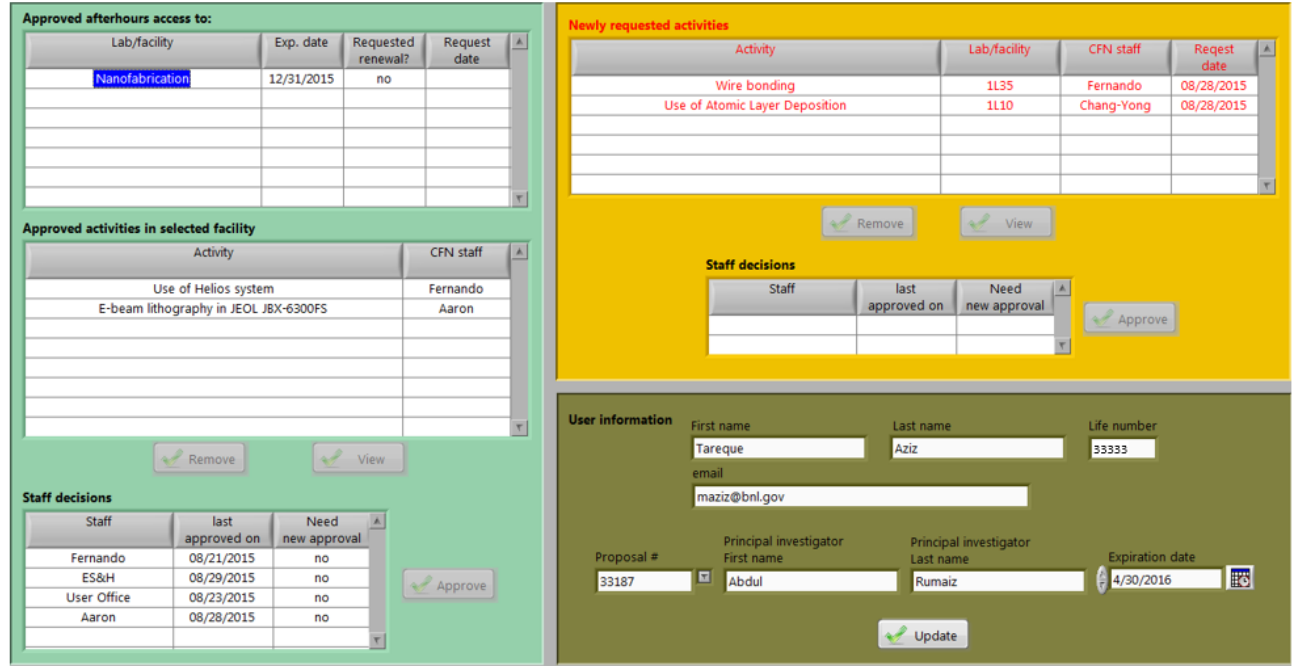

Figure 14. Approval of new activity from an active facility. (a) New activities (yellow) and facility access (partial view, light green) sections of the review afterhours request module seen by the ES\&H staff before clicking the "Approve" button. Notice that the facility of the selected activity is listed in the facility access section. In this case, this user already has access to the nanofabrication facility. (b) Same sections of the program after the ES\&H staff has clicked the "Approve" button. 
- Steps 8 and 9 (Facility is not listed): add facility (but set as expired), and email Users Office staff. In Fig. 15a, the selected activity's facility, lab 1L35, is not listed in the facility access section for this user. Once the ES\&H staff approves the request, the activity is erased from the new activities table and the facility and activity are added to the facility access section (see Fig. 15b). The facility shows as expired (red color), because the Users Office staff approval is set to pending. Subsequently, an email is sent to the Users Office staff to request activation of the user's badge for afterhours access to the facility.

- Steps 10 and 11: collect Users Office staff approval and set facility access active. These final steps are identical to Steps 7 and 8 in Section 3.2.1.

a)

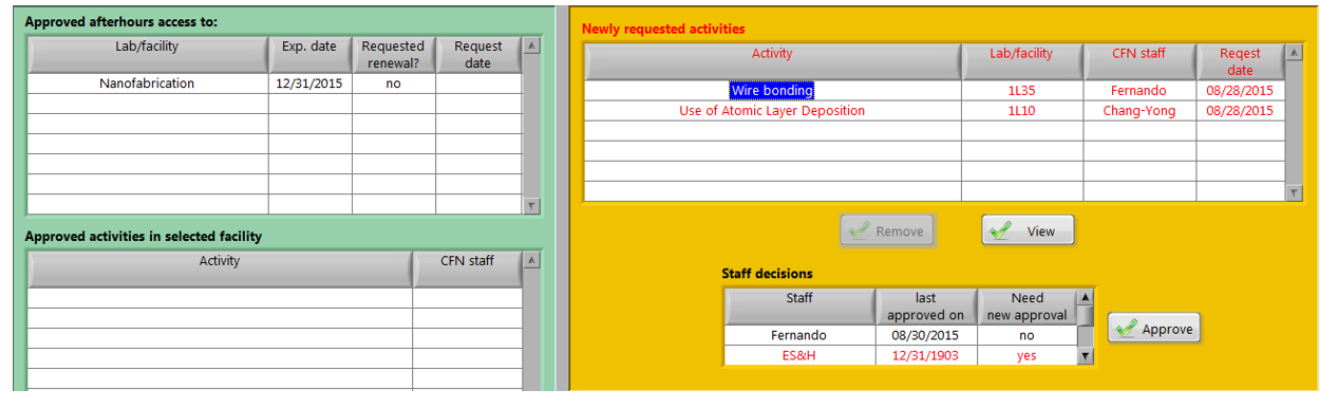

b)

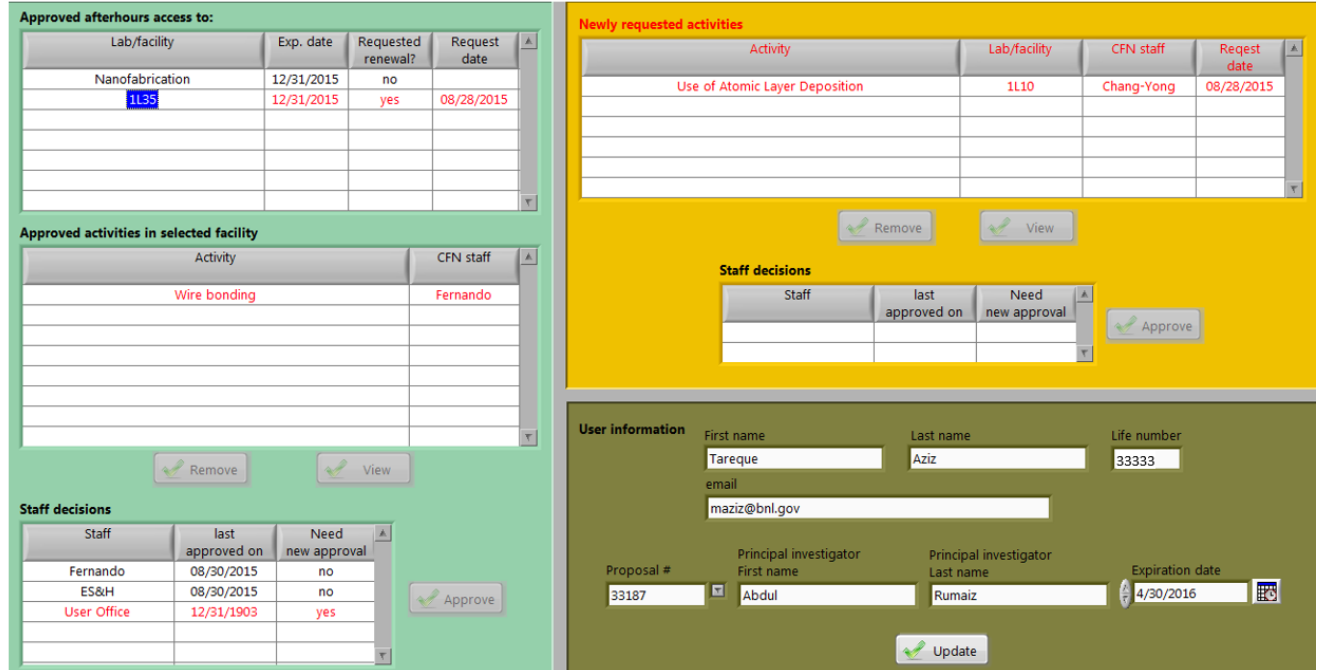

Figure 15. Approval of new afterhours activity request from a facility not listed in the user's list of accessible facilities. (a) New activities (yellow) and facility access (light green) sections of the review afterhours request module seen by the ES\&H staff before approving the request. Notice that lab 1L35 is not listed in the facility access section. (b) Once approved by the ES\&H staff, the facility and activity are added to the facility section, but in red color (expired), because the Users Office approval is set to pending.

\subsection{The edit afterhours activities module}

The edit afterhours module, shown in Fig. 16, is launched when a staff member clicks on the "Edit afterhours activities" button in the staff review section of the main user interface (Fig. 1). It allows staff to edit or add new activities to the afterhours activity database. The facility activity list, in the bottom left area of Fig. 16, contains all the activities available in the facility selected in the top left list (purple area). The right area of Fig. 16 displays the activity form of the selected activity. Only the staff owning the selected activity and the ES\&H staff can edit an activity. Setting an activity's status to "Inactive" (top entry in the activity form) removes it from the users' list of permitted activities. Any 
staff can add a new activity in a selected facility by clicking on "ADD" (the first element of the activity list) and filling out the fields of the activity form.

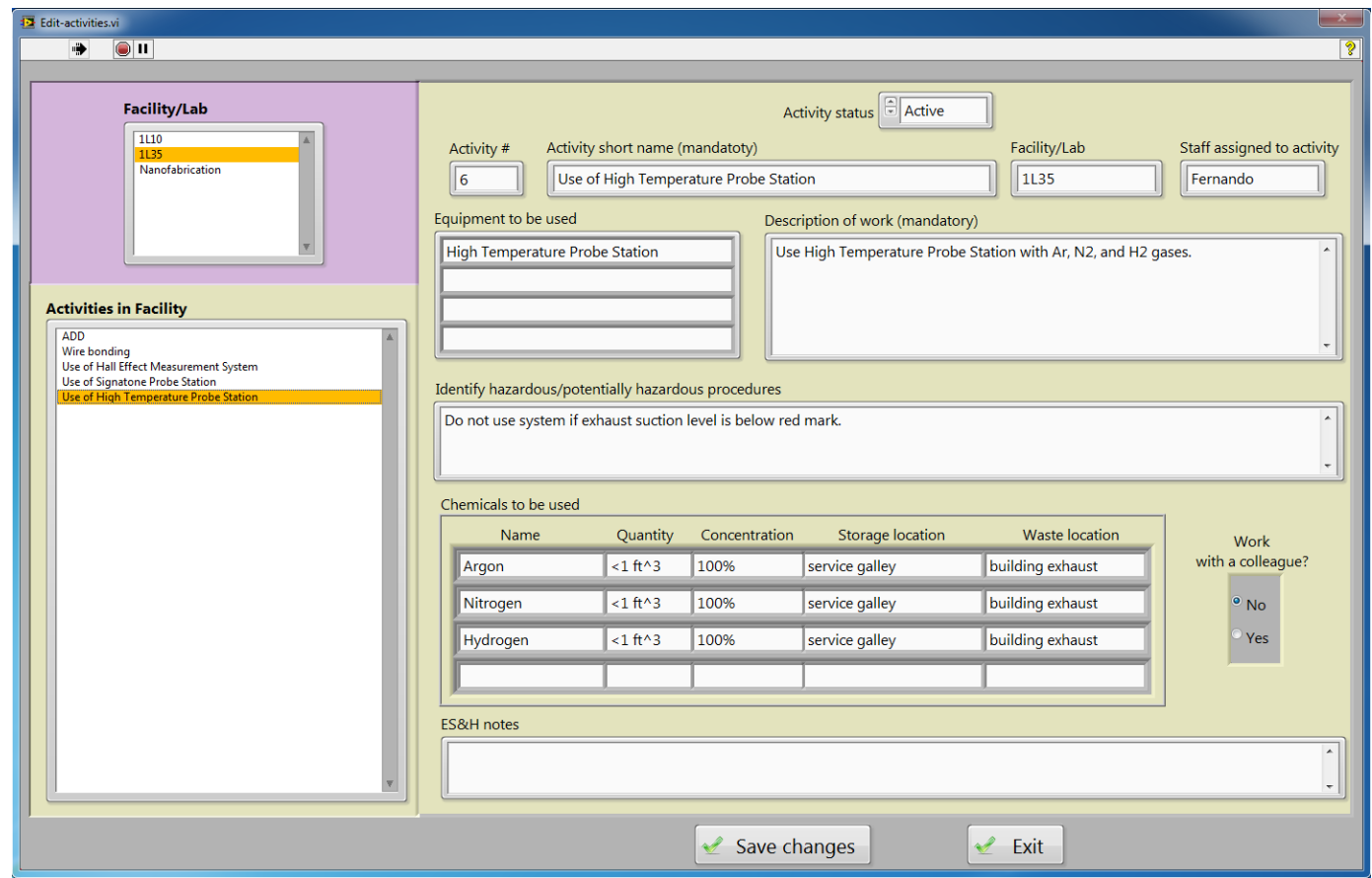

Figure 16. User interface of the edit afterhours activity module.

\section{Discussion}

From an administrative point of view, as described in the previous sections, the afterhours software benefits users and staff in a facility by simplifying and automatizing the afterhours request and approval process. From a safety point of view, it informs users (and staff) of the specific afterhours activities they are allowed to perform. It is true that by itself, the afterhours software is not an engineering safety control that can physically prevent users from accessing labs or performing activities not listed in their list of permitted activities. However, the afterhours software can interact with physical safety mechanisms found in user facilities, to become a powerful engineering safety control.

At a first level of additional safety, individual labs in a user facility can implement a lab login software, ${ }^{3}$ which, among other features, ${ }^{7}$ keeps track of who is entering the lab. The login software can easily share information with the afterhours software and block access to users who do not have afterhours access to that facility.

At a higher safety level, in some facilities, like the Center of Functional Nanomaterials, electronic door controllers provide access to facilities upon positive authentication of users' ID badges. It is possible to pass information from the afterhours software to the ID badging system in order to physically block users from entering facilities afterhours.

An even higher level of safety can be provided when the afterhours program shares information with a facility instrument access control system, which uses a hardware control to block users from accessing a particular instrument. The afterhours software is designed to interact with this kind of control system. As shown in Section 3, each afterhours activity possesses and activity form (Figs. 3 and 16), which contains a list of the instruments necessary for the activity. Before allowing user access to an instrument, the instrument control system can query the afterhours software in order to verify that the instrument is listed in one of the user's active afterhours activity forms. As an added benefit of this integration, users can report problems to staff during afterhours work by means of the feedback feature usually found in most instrument control systems. 


\section{Conclusions}

The afterhours management program for shared facilities presented here handles the processes required for afterhours access (request, approval, extension, etc.) and automatizes the creation and maintenance of an afterhours database. The program implements the concept of permitted afterhours activities, which establishes a well-defined list of activities that users can perform afterhours. Administratively, the program handles diverse tasks which end up saving valuable time for users and staff. With respect to safety, its main contribution is to offer users an easy-to-access platform where they can view their permitted list of afterhours activities.

The concept of permitted afterhours activities is a safety administrative control, whose efficacy relies on the users' compliance with good laboratory practices. However, the safety aspect of the program can be enhanced further, even to include engineering controls, by merging it with other safety systems like lab door and instrument access control systems, which are becoming standard in most user facilities.

\section{Acknowledgements}

This research used resources of the Center for Functional Nanomaterials, which is a U.S. DOE Office of Science Facility, at Brookhaven National Laboratory under Contract No. DE-SC0012704. The authors are thankful to Dr. Charles Black and Dr. James Dickerson for their support in implementing the software in the Center for Functional Nanomaterials.

\section{Annotations}

[1] The afterhours activities management software was developed using LabVIEW 2014 Base System. Following a request by the authors for DOE approval, the code may be made available as open source.

[2] Statistical data reported in DOE's Triennial Review of the CFN Operations and Research conducted in August, 2016. A complete list of CFN's instrumentation and facilities can be accessed at https://www.bnl.gov/cfn/.

[3] The facility login program performs several functions for specific facilities of the Center for Functional Nanomaterials. In particular, it maintains a database of users, proposals and proposal expiration dates. A valid user must be listed as a member of an unexpired proposal. This program is written using the same programming language as the afterhours software.

[4] The concept of permitted afterhours activities is based on the similar concept of permitted chemical activities, which is used since 2010 by a software for directing chemical processes in the nanofabrication facility of the Center for Functional Nanomaterials.

[5] The Request new activity module only lists activities from active facilities (black font in Fig. 2a) or from facilities that the user does not currently have access.

[6] In the Center for Functional Nanomaterials, door access to the main building and individual labs is controlled by an electronic badge reader system.

[7] Two of the most popular safety features of the facility login program are: 1) It blocks users who have been absent from the lab for more than a determined amount of days, and 2) it presents at $\log$ on a safety quiz question that users need to respond correctly in order to access the lab. 